\title{
Separation Assurance and Scheduling Coordination in the Arrival Environment
}

\author{
Arwa S. Aweiss ${ }^{1}$, Andrew C. Cone ${ }^{2}$, Joshua J. Holladay ${ }^{3}$, Epifanio Munoz ${ }^{4}$ \\ NASA Ames Research Center, Moffett Field, CA, 94035 \\ Timothy A. Lewis 5 \\ NASA Langley Research Center, Hampton, VA, 23681
}

\begin{abstract}
Separation assurance (SA) automation has been proposed as either a ground-based or airborne paradigm. The arrival environment is complex because aircraft are being sequenced and spaced to the arrival fix. This paper examines the effect of the allocation of the SA and scheduling functions on the performance of the system. Two coordination configurations between an SA and an arrival management system are tested using both ground and airborne implementations. All configurations have a conflict detection and resolution (CD\&R) system and either an integrated or separated scheduler. Performance metrics are presented for the ground and airborne systems based on arrival traffic headed to Dallas/ Fort Worth International airport. The total delay, time-spacing conformance, and schedule conformance are used to measure efficiency. The goal of the analysis is to use the metrics to identify performance differences between the configurations that are based on different function allocations. A surveillance range limitation of $100 \mathbf{~ n m i}$ and a time delay for sharing updated trajectory intent of 30 seconds were implemented for the airborne system. Overall, these results indicate that the surveillance range and the sharing of trajectories and aircraft schedules are important factors in determining the efficiency of an airborne arrival management system. These parameters are not relevant to the ground-based system as modeled for this study because it has instantaneous access to all aircraft trajectories and intent. Creating a schedule external to the CD\&R and the scheduling conformance system was seen to reduce total delays for the airborne system, and had a minor effect on the ground-based system. The effect of an external scheduler on other metrics was mixed.
\end{abstract}

\section{Nomenclature}

ACES $=$ Airspace Concept Evaluation System

ADS-B = Automatic Dependent Surveillance Broadcast

ARTCC $=$ Air Route Traffic Control Centers

ATA $=$ Actual Time of Arrival

ATM $=$ Air Traffic Management

$\mathrm{CDF}=$ Cumulative Distribution Function

${ }^{1}$ Aerospace Engineer, Aviation Systems Division, M/S 210-10, AIAA Senior Member.

${ }^{2}$ Aerospace Engineer, Aviation Systems Division, M/S 210-10, AIAA Member.

${ }^{3}$ System Analyst, University of California Santa Cruz, Aviation Systems Division, M/S 210-10.

${ }^{4}$ Aerospace Engineer, Aviation Systems Division, M/S 210-10.

${ }^{5}$ Sub-Project Manager, Crew Systems and Aviation Operations Branch, M/S 152, AIAA Member. 


$\begin{array}{ll}\text { CD } & =\text { Conflict Detection } \\ \text { CR } & =\text { Conflict Resolution } \\ \text { CD\&R } & =\text { Conflict Detection and Resolution } \\ \text { FA } & =\text { Function Allocation } \\ \text { KDFW } & =\text { Dallas/Fort Worth Airport } \\ \text { MOPS } & =\text { Minimum Operational Performance Standards } \\ \text { NAS } & =\text { National Airspace System } \\ \text { NextGen } & =\text { Next-Generation Air Transportation System } \\ \text { SA } & =\text { Separation Assurance } \\ \text { STA } & =\text { Scheduled Time of Arrival } \\ \text { TBFM } & =\text { Time-based Flow Management } \\ \text { TFM } & =\text { Traffic Flow Management } \\ \text { TMA } & =\text { Traffic Management Advisor } \\ \text { TRACON } & =\text { Terminal Radar Approach Control Facility }\end{array}$

\section{Introduction}

A ir traffic controllers provide separation assurance and other services to aircraft operating under Iinstrument flight rules (IFR) to enable the safe, orderly, and expeditious flow of air traffic. However, this human-centric ground-based system is reaching a limit to the number of aircraft that can be safely managed at any given time, presenting a chokepoint for international plans to accommodate growth in demand for air transportation.

New methods for providing separation assurance are needed in order to enable significant advances in air traffic capacity and efficiency while preserving the safety of the system. Automation is believed to be part of a solution that will help meet the projected increase in the level of air traffic. Function allocation, the effective allocation of separation assurance functions to humans and automation systems in the air and on the ground, is one aspect of separation assurance research. In order to accommodate significant growth in demand for air transportation, this allocation must evolve and adapt to new requirements through changes in automation levels and in the roles and responsibilities of controllers and pilots. Function allocation is a major issue in the evolution of the current separation assurance system as well as the design of its successor. Researchers have been studying two largely different ways (ground-based and airborne) to provide separation assurance. For example, a ground-based concept called the Advanced Airspace Concept $(\mathrm{AAC})^{1}$ was originally designed to reduce controller workload by increasing automation and potentially replacing the existing system. The Distributed Air/Ground Traffic Management concept (DAG-TM) was a set of concept elements that attempted to provide an approach that can allow more user flexibility and increased capacity in a specific phase of flight and operational domain. ${ }^{2}$ Autonomous Flight Rules (AFR), an airborne separation assurance concept, proposes to transfer responsibility for separation assurance to the pilot on an individual aircraft basis. ${ }^{3}$

An important aspect of separation assurance (SA) function allocation research is to develop an understanding of the interaction between traffic management systems. A situation where coordination between systems might be necessary is the arrival environment. Arrival aircraft are routed through specific spatial locations known as arrival fixes that serve as entry points to the Terminal Radar Approach Control (TRACON). During arrival merging and spacing, creating arrival schedules independently from safe and flyable trajectories may result in either infeasible or overly conservative schedules. Function allocation (FA) plays a role in the design of an air traffic management (ATM) system. The arrival environment is a complex situation and is an important part of an ATM system. Therefore, the arrival-merging situation will be analyzed in this study.

This paper presents results from a fast-time air traffic simulation with analysis of "ground-inspired" and "airborne-inspired" separation assurance coordination schemes alongside two approaches to arrival scheduling. For the experiments in this study, these are considered different levels of coordination. One level is a system that jointly solves separation conflicts in conjunction with sequencing and spacing to maintain an arrival schedule. In contrast, another level is a system that decouples these functions and solves them independently. Results of the performance of these for both a ground-based and airborne system are presented in this paper. It is important to note that choosing a best option is not a goal of this study. These results are intended to identify the strengths and weaknesses of each configuration and to point to features that would need to be further investigated or developed to move towards an operational system. 
The paper is organized as follows. Section 2 provides background information on separation assurance in relation to the problem of function allocation. Section 3 describes the methodology for the present study, which includes a system description and tools used, experiment description and setup, and metrics. The results are presented in Section 4. In the last section, a conclusion highlighting the key findings of this work is presented.

\section{Background}

\section{A. Function Allocation and Separation Assurance}

A separation assurance system in air traffic management performs the functions necessary to ensure that aircraft are safely separated from other traffic and also deals with the avoidance of severe weather. It is also responsible for the conformance of flights to arrival constraints, which helps in providing expeditious flow of traffic from origin to destination. Separation Assurance Function Allocation has traditionally been conceptualized along two axes. The first is the Ground-Air axis, which can be thought of as a locus of function control; this investigates what functions and under what conditions should these be performed by a central authority (ground-based separation) or distributed among individual aircraft (airborne separation). The second axis is Human-Automation, which investigates which functions should be performed by human operators and which should be performed by automation systems and under what conditions. Along this axis lie many questions related to the design of human-machine interfaces and the limits of both humans and software algorithms in safety-critical roles. ${ }^{4-11}$

Today's SA services are provided largely by ground-based and human-centric systems. There exists a need to modernize this system in order to meet the growing demands for air travel and accommodate new airspace uses in a safe, efficient, and sustainable manner. Although there has been research on both groundbased and airborne separation assurance systems, the future SA system is currently not well defined. This led to the idea of examining function allocation in regards to separation assurance. Function allocation research is intended to help better understand the tradeoffs associated with varying the locus of control and introducing greater levels of automation into air traffic operations.

\section{B. Function Allocation and Arrival Management}

The purpose of arrival traffic management is to maintain an efficient flow of traffic through the airspace to meet airport capacities or arrival rates. Time-based metering is one means of efficiently sequencing and spacing arrival traffic at rates conforming to the maximum arrival rate for a given airport. ${ }^{12}$ Arrival aircraft are routed through specific spatial locations known as arrival fixes that serve as entry points to the Terminal Radar Approach Control (TRACON). TBFM (Time-based Flow Management) uses time-based as opposed to distance-based metering to help controllers separate air traffic. It uses the capabilities of the Traffic Management Advisor (TMA), which is a system that is already deployed to all en route centers. ${ }^{13}$ TMA was developed to create schedules based on predicted times of arrival and was not intended to generate a conflict-free trajectory nor a fuel-efficient trajectory. ${ }^{14,15}$

Arrival Management is an important aspect of the Next-Generation Air Transportation System (NextGen) and an essential component of an SA system. A key issue of function allocation in the arrival environment is the level of coordination between the agent(s) performing separation assurance (i.e., resolving traffic conflicts) and the agent(s) performing arrival management (i.e., scheduling, spacing, and sequencing). The particular approach to separation assurance may impact the ability to effectively organize and condition traffic for arrival into complex terminal airspace.

Time constraints at a single point -such as are enforced with time-based metering- may not be sufficient to ensure safe separation and time conformance of aircraft as they approach the airport since aircraft approach from different directions, altitudes, and airspeeds. In fact, the presence of overflights and other arrivals maneuvering in the airspace can make it difficult to find a conflict-free trajectory that meets a desired time of arrival at a target arrival fix. This causes extra delays that can impact the rest of the schedule by forcing a number of upstream aircraft to take more delay than they would have if the first aircraft had been able to meet its original target time. Thus, tighter coordination of the separation assurance and time-based scheduling functions may be required for certain concepts to operate efficiently under certain constraints, while in others they may be able to operate efficiently with less coordination. Understanding this relationship is important because the level of coordination necessary between the 
separation assurance and arrival management components could affect the safety and efficiency of the overall system in different ways. This study investigates some of these effects for such systems.

Integration of arrival management with an external ground-based conflict detection and resolution system has been explored in previous studies. Aweiss et al. ${ }^{16}$ discussed the implementation of a groundbased separation assurance system that performed both conflict detection and resolution and arrival management. One component of the separation assurance system provided conflict probing for both arrivals and non-arrivals and maneuvers to resolve conflicts for non-arrival flights. The other component performed arrival scheduling, sequencing, and provided maneuvers to resolve conflicts for arrivals to a single fix. Results indicated that the integrated system reduced the number and magnitude of time-based spacing violations without adversely affecting the resolution success rate of the system. In a 2012 paper ${ }^{17}$, results of an evaluation of the performance of an existing arrival scheduler and a prototype resolution generator that were integrated to provide multiple-fix metering were presented. Results indicated that arrival fix and airport configurations might play a role in system performance and that during high traffic periods, tighter coupling of the scheduling and resolution generation system may improve performance.

\section{Methodology}

\section{A. System Description}

This section describes the system used in the experiment. The components responsible for creating a schedule, conforming to a schedule, and maneuvering aircraft are described. The simulation platform, the airspace examined, and the rules of the airborne implementation are also discussed.

Since the research focus is separation assurance function allocation in the arrival environment, the main agent functions are arrival scheduling, scheduling conformance, and conflict detection (CD), and conflict resolution (CR) for distance-based separation. $\mathrm{CD}$ for distance-based separation involves detecting conflicts that would violate the distance-based separation requirements and CR is finding a maneuver that will prevent a distance-based conflict. Schedule conformance involves comparing an aircraft's scheduled time of arrival (STA) to its currently estimated arrival-fix crossing time and finding a conflict-free maneuver that will allow the aircraft to conform to its given schedule. For the purpose of this study, simulation agents use Autoresolver, a component of the Advanced Airspace Concept (AAC) ${ }^{18}$, to handle these functions. Autoresolver maintains aircraft separation from other aircraft, from hazardous weather, and from restricted airspace. ${ }^{19}$ It can also provide trajectories to meet time-spacing constraints that are provided by either an external system or its own integrated scheduler described below. ${ }^{20}$ Autoresolver is not restricted to a specific concept of operations and can be used for airborne and ground-based systems. The systems presented in the remainder of this paper will be referred to as a CD\&R and scheduling conformance system.

There are two scheduling algorithms used in this experiment. The first is an integrated scheduler, and the second is a separated scheduler. Both are first-come, first-served algorithms that schedule to each arrival fix independently. For the cases with the integrated scheduler, a single system is allocated responsibility for the scheduling, $\mathrm{CD} \& \mathrm{R}$, and scheduling conformance functions. In the separated scheduler cases, a disparate system, similar to TMA, is allocated responsibility for the scheduling function. It sends the scheduled times of arrival (STAs) to the separate system that is responsible for the CD\&R and scheduling conformance functions.

In this study, an existing air-traffic simulation system known as the Airspace Concept Evaluation System (ACES) is used as the simulation platform. ${ }^{21}$ ACES is a multi-fidelity, non-real-time modeling and simulation system with full gate-to-gate representation of all the major components of the NAS. The agentbased modeling approach that is being used represents the individual behaviors and functions of the airspace participants. These autonomous agents act on their own best interests using decision-making rules and information available to them. For the ground-based implementation, the airspace centers (e.g. Cleveland (ZOB) ARTCC, Fort Worth (ZFW) ARTCC, etc.) act as individual agents. Each groundmaintained aircraft belongs to a center agent, and the center is the sole decision maker. The airborne implementation allows each aircraft to act as an independent agent throughout the airspace.

There are several inherent differences that arise from placing the separation assurance responsibility with the aircraft versus the ground. Autoresolver is allocated responsibility for the CD\&R and schedule conformance functions in all the simulations. Therefore, the two differences that have the biggest impact on system behavior are likely to be surveillance limitations and coordination rules between aircraft. The ground-based agents have surveillance within the center boundary or near the center boundary. Airborne 
agents detect other aircraft within a fixed horizontal range, forming a disk-shaped "surveillance" region around the agent. Autoresolver is used in all the simulations to control these variables. A $100-\mathrm{nmi}$ surveillance range was used in these experiments based on detailed ADS-B model and Class A3 Minimum Operational Performance Standards (MOPS) requirements. ${ }^{22,23}$

When two airborne agents are in conflict, right-of-way rules determine which aircraft has priority while the other is burdened with the responsibility of resolving the conflict. Scheduled aircraft have priority and non-scheduled aircraft must resolve the conflict. If both aircraft are scheduled or neither is scheduled, a "coin-flip" algorithm gives one aircraft priority with 50\% probability. Both aircraft know the outcome of the algorithm; therefore aircraft priority is implicitly coordinated. Also, for airborne systems, the simulation currently assumes that an aircraft can only maneuver itself. More details can be found in Holladay et al. ${ }^{21}$

\section{B. Arrival Sequencing Description}

Figure 1 is a depiction of arrival scheduling. The arrival fix is a point in space at which aircraft transition from Air Route Traffic Control Centers (ARTCC) to TRACON airspace. The first time an aircraft gets within 20 minutes of its arrival fix, it is considered an arrival. The freeze horizon specifies the time horizon, as measured from the arrival fix, at which arrival aircraft become eligible for scheduling and generation of maneuvers to meet the schedule. The freeze horizon was set to 20 minutes so that inbound arrival aircraft would still be in their cruise phase of flight; this is consistent with the freeze horizon used in prior studies. ${ }^{16,19,20,24-26}$ For this study, the time-based separation requirement for arrival scheduling was modeled as a time-spacing constraint, which sets a lower bound on the interval between every two consecutive aircraft, Aircraft A and B for example, that cross the same arrival fix. A scheduler with a firstcome-first served algorithm is used. When being scheduled, an aircraft at the freeze horizon is not concerned with upstream aircraft. In this figure, Aircraft D is predicted to be in violation of the timespacing constraint with aircraft $\mathrm{C}$ at the arrival fix; therefore, Aircraft D must be issued a delayed STA. This means that a new trajectory for Aircraft D, that meets both the distance-based and time-based separation requirements, needs to be generated.

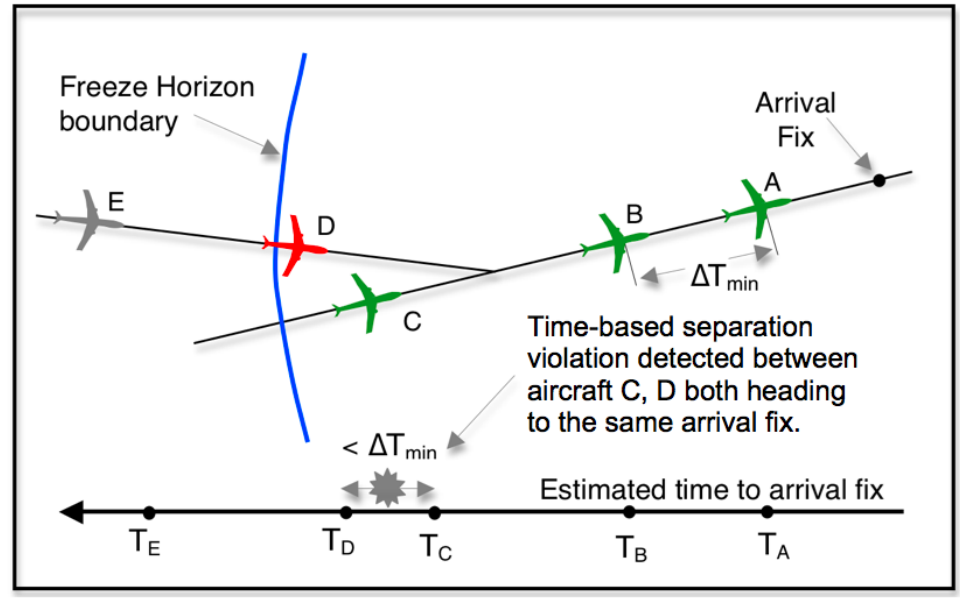

Figure 1. Arrival scheduling

\section{Experiment Description}

This study on coordination between SA and arrival management is divided into two configurations: the integrated system and the separated system. Each configuration had a ground-based and an airborne implementation (Fig. 2). The following is a more detailed description of the configurations:

1) Integrated System

This is referred to as an "integrated system"3 since the same system is allocated responsibility for CD\&R, arrival scheduling, and scheduling conformance. This system checks the trajectories of aircraft as they cross the freeze horizon and decides whether an aircraft may proceed along its current trajectory, or if it needs to be maneuvered to avoid violating a distance or time-based constraint at the

\footnotetext{
${ }^{3}$ The "integrated system" described in this paper differs from the integrated system as defined in Reference 13.
} 
arrival fix. If the aircraft needs a new arrival fix time, the system can determine a new arrival fix crossing time and send a maneuver to the aircraft that is conflict free and meets that new time. The schedule is maintained within the system and the scheduler can alter the aircraft's schedule if it cannot meet the desired arrival-fix crossing time. This is said to have feedback since the schedule is being developed alongside trajectory generation within the system and is constantly being updated with an aircraft's currently predicted time of arrival. In this study, the ground-integrated system is considered to have a high level of coordination. The ground system immediately knows the new trajectories and STAs of each aircraft at the arrival fix. An airborne system has a partial level of coordination because the aircraft are responsible for their own CD\&R and scheduling. In an airborne system, when an arrival aircraft reaches the freeze horizon it needs to broadcast its STA and the trajectory it generates to meet that STA, to the other arrivals. In these experiments, other arrivals cannot adjust their schedule until the next conflict-detection cycle when they are able to receive this information. This creates a communication delay, which does not exist in the ground system. For both the ground-based and airborne systems, trajectories are shared without errors and with full knowledge of intent.

\section{2) Separated System}

This is referred to as a "separated system" since responsibility for CD\&R and scheduling conformance are allocated to one system, while generating schedules is allocated to a second system. A scheduler based on the TMA concept was used in this experiment. In this system, a first-come-firstserved scheduler generates a schedule for all aircraft as they approach the freeze horizon. When an aircraft crosses the freeze horizon, the CD\&R and conformance system checks the schedule and compares the aircraft's currently estimated arrival-fix crossing time with the time assigned by the scheduler. If there is a difference, the CD\&R and conformance system, will try to find a conflict-free maneuver that will meet that assigned time. This configuration has a partial level of coordination for both ground and airborne systems. This is because in this system, the schedule is maintained separately from the CD\&R and conformance system logic and is not updated if aircraft cannot find a maneuver that conforms to their target time. In other words, if something occurs during arrival that forces an aircraft to either maneuver to avoid a predicted conflict or the aircraft was unable to find a maneuver that would meet the arrival time, the external scheduler will continue scheduling aircraft, assuming all downstream aircraft met their assigned times. As in the integrated system, the schedule and trajectory information is known by the ground system immediately while for the airborne, the aircraft need to broadcast their information and there is a communication delay.

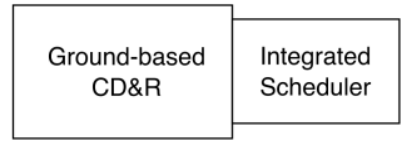

Ground CD\&R with

integrated scheduling

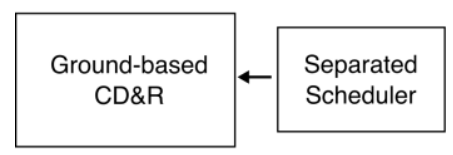

Ground CD\&R with

separated scheduling

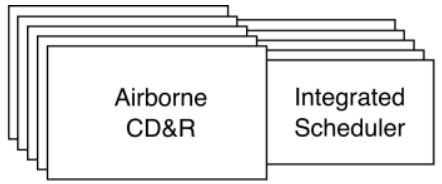

Airborne CD\&R with integrated scheduling

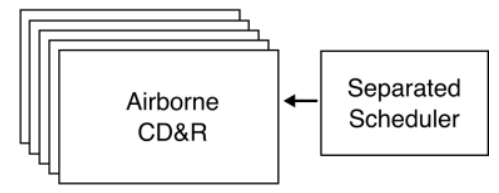

Airborne CD\&R with separated scheduling

Figure 2. Ground-based and airborne system configurations

\section{Experiment Setup}

The experiment uses realistic traffic scenarios that were developed from actual filed flight plans of aircraft from low-weather-impact days randomly selected from 2012. The first day is April 6, 2012, which consists of 3,207 flights passing through ZFW Center, 578 of which are arrivals headed to Dallas/Fort 
Worth (KDFW) Airport. The second day is October 7, 2012, with a total of 3,312 flights, 658 of which are arrivals and the third is October 16, 2012 with a total of 3,521 flights, 706 of which are arrivals. A simplified configuration of the KDFW airport consisting of only four arrival fixes was used. Each arrival aircraft is going to one of four fixes located in the Northeast, Northwest, Southeast, and Southwest corners of the TRACON, and each fix is sequenced independently. The scenarios were simulated using ACES. No trajectory prediction errors or weather were modeled in order to establish a baseline and allow for an assessment of the independent variables. Both the integrated and the separated scheduler enforced a minimum of 72-second spacing.

\section{E. Metrics}

The primary metrics examined in this study can be grouped into categories of efficiency and safety. To study efficiency, the rate at which aircraft cross the arrival fix, the scheduling conformance, and total delay for arrival aircraft were examined. These metrics were used to compare the different test cases and highlight the differences.

The rate at which aircraft pass through the arrival fix is measured by the consecutive actual times of arrival (ATA) metric. This measures the time-spacing between aircraft as they cross the arrival fix and head into the Terminal area. In this study, this metric is filtered to only include aircraft that were maneuvered to meet a schedule. This excludes aircraft that have more than the required time-spacing before being scheduled. This metric can be used to check how well the system is meeting the target spacing for aircraft that need to be scheduled. Differences in this metric imply different scheduling characteristics or restrictions, or that different resolution strategies are being used.

Schedule conformance is a measure of the difference between the scheduled time of arrival (STA) and the actual time of arrival (ATA). The STA is generally evaluated at the freeze horizon, while the ATA is recorded as an aircraft crosses the arrival fix. This metric gives an indication of how well the scheduling conformance algorithm met the target time-spacing. Possible reasons for extra spacing would include aircraft being forced to maneuver to avoid a conflict after being scheduled, and not being able to find a maneuver that meets the target spacing. For this study, this metric is only relevant to the separated system.

Figure 3 shows two diagrams depicting examples of when an aircraft is required to find a new maneuver, and possibly a new time slot, in order to maintain separation down to the arrival fix. In Figure 3a, Aircraft C and D are approaching the same arrival fix. In an airborne concept, it is possible that aircraft arrive at the freeze horizon at the same time and independently generate maneuvers to meet their schedules simultaneously. Downstream they realize there is a potential conflict and one of them needs to generate a new maneuver. Figure $3 b$ depicts two aircraft approaching the same fix at about the same time. Here, aircraft A and aircraft B are separated by more than $100 \mathrm{nmi}$. When Aircraft A reaches the freeze horizon it is unable to detect Aircraft B because it is not within its surveillance range and therefore executes a maneuver that results in a distance-based or time-based conflict. Such instances can make it harder for the aircraft to meet its STA. There will be consequences independent of the concept of operation used. For example, rescheduling an aircraft could cause delay to propagate upstream. However, the amount of delay and the aircraft absorbing delay is dependent on the concept used. The ground-based system is able to detect both aircraft and issue maneuvers accordingly and thus aircraft should not have to maneuver again after the freeze horizon. 


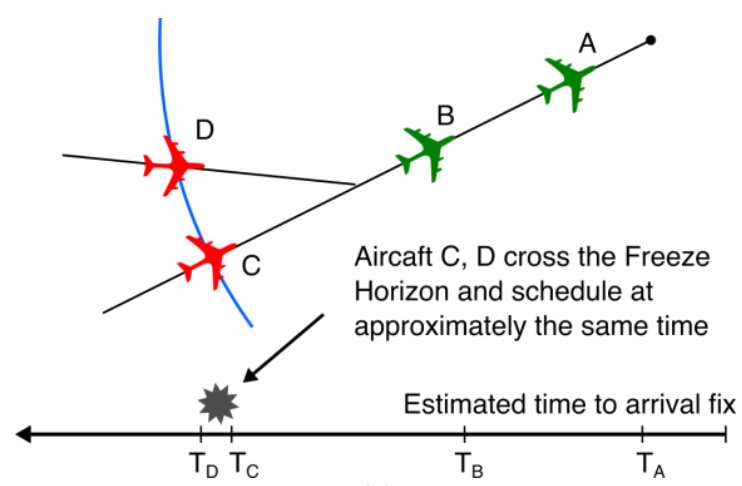

(a)

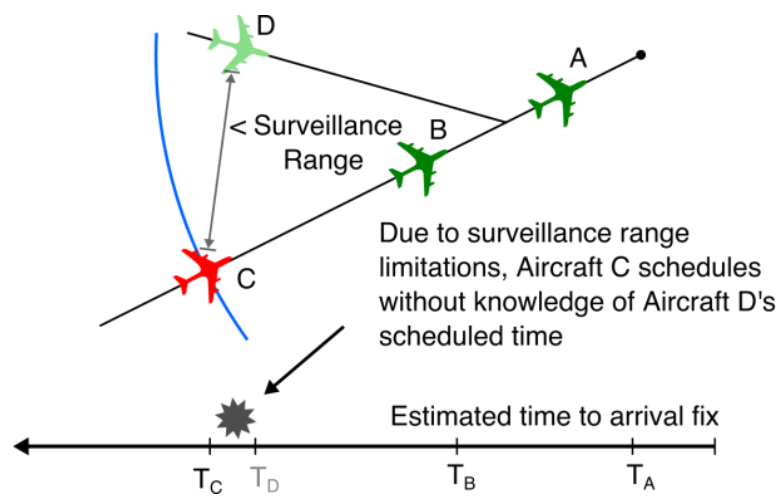

(b)

Figure 3. Maneuvering after freeze horizon (a) Simultaneous scheduling (b) Surveillance

The last efficiency metric examined in this study is delay. First, the total delay assigned at the freeze horizon for all arrivals is presented. Then a comparison between the delay requested by the separated scheduler versus the delay assigned by the agent responsible for scheduling conformance is presented. The total delay is used to highlight some differences between airborne and ground implementations in this simulation. The requested versus actual delay can give some insight into where any extra delay is being accrued.

The number of losses of separation (LOS) is usually used as a metric for safety. A loss of separation occurs when two aircraft pass within 5 nautical miles horizontally and 1000 feet vertically of each other. There were no LOS cases for arrival aircraft in these experiments. Errors in the predicted intent or future state of an aircraft would be the primary reasons LOS cases would occur in an automated system. However, there is no trajectory prediction error in this simulation, and trajectory intent, when shared, is perfect. This is because both of these parameters could not be realistically addressed in the scope of this work. Therefore, this study will use the "near ideal" case in regards to both the trajectory intent quality and trajectory prediction accuracy. It is still possible to get LOS cases if the automated system has more aircraft than it is capable of dealing with, or if there is a predicted conflict with no possible resolution. Neither of those situations occurred in these experiments. For airborne systems, it is also possible for a LOS to occur if two aircraft maneuver before they have had a chance to share their new intent. That situation also did not occur, but it is one to address when discussing airborne systems. 


\section{Results}

The results of the experiments are presented in this section. All tables and figures shown are based on aggregate results from three different days of traffic from 2012. Aggregate values are presented because the results across the three days were qualitatively similar.

Figure 4a shows the consecutive ATAs at the arrival fixes for all arrivals in the separated system. This figure shows that overall the airborne systems have more time-spacing between aircraft. This lines up with other results that will be presented later. Effectively, airborne systems are more likely to need to adjust an aircraft after the freeze horizon due to a trajectory that was not accounted for either because the other aircraft was out of surveillance range, or because two aircraft maneuvered to meet a schedule at the same time step. Again, both of these situations can require an aircraft to maneuver again after the freeze horizon.

Figure $4 \mathrm{~b}$ is a similar plot for the integrated system. The results show this system stays close to the 72 second target spacing for a longer duration than in the separated system. The two curves are close in both cases, though there is a higher percentage of arrivals close to the target of 72 seconds for the ground system. This is expected given the reduced surveillance range and level of coordination the airborne system has in these simulations. Overall, all the simulation configurations have approximately $70 \%$ of aircraft close to the target spacing of 72 seconds. These trends, as well as reasons for why the airborne cases have more difficulty meeting the target spacing, will be explained in the rest of the results section.

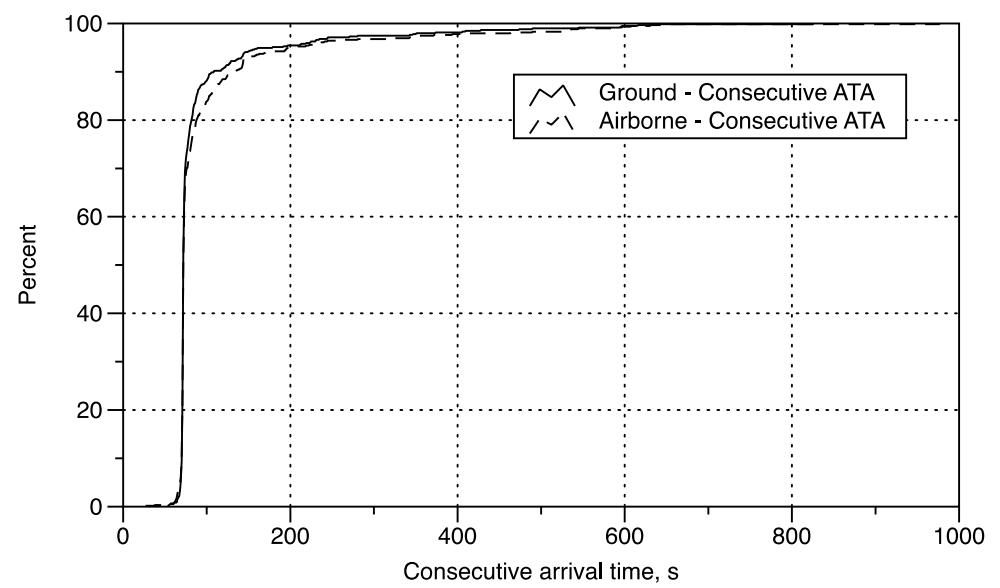

(a)

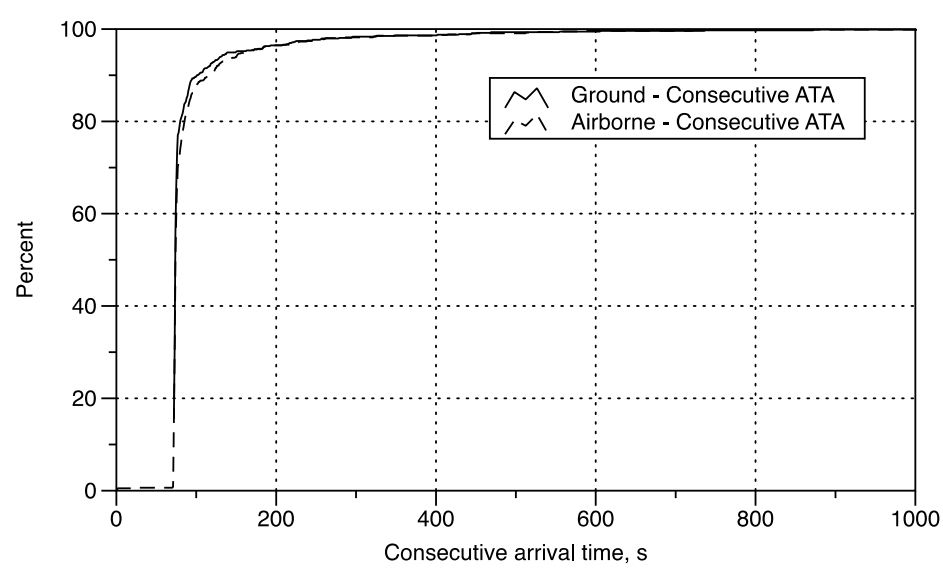

(b)

Figure 4. CDF of Consecutive Arrival Fix Time (a) Separated system (b) Integrated system 
Figures $5 \mathrm{a}$ and $5 \mathrm{~b}$ are CDFs of the separated scheduler conformance at the freeze horizon and arrival fix, respectively, aggregated across the three days. The grey bar in each diagram represents a 10-second window in which aircraft are considered to have conformed to their schedules. Figure $5 \mathrm{a}$ is the conformance at the freeze horizon. This is based on the predicted conformance at the time when an aircraft is being scheduled. The overall curves for the ground and airborne cases are similar, though there are slight differences at specific points. For example, 83\% of arrival aircraft are within 10 seconds of their scheduled time of arrival when the ground system is responsible for the scheduling and $85 \%$ when the airborne system is responsible for the scheduling. Figure $5 \mathrm{~b}$ is a CDF of predicted conformance at the arrival fix. The percentage of the aircraft in conformance is still $83 \%$ for the ground system, while the percentage is $80 \%$ for the airborne system, slightly less than the $85 \%$ predicted conformance at the freeze horizon. The reason the airborne system has a decrease in conformance when moving from the freeze horizon to the arrival fix is due to arrivals that are forced to reschedule after the freeze horizon. These reschedules are necessary to maintain a minimum time-spacing between arrivals and/or to avoid a distance-based conflict with another arrival. The reason this only occurs in airborne systems in these simulations is because the ground systems do not have the limited surveillance range or the simultaneous scheduling features that exist in the airborne systems. In order for an airborne system to maintain the levels of schedule conformance attainable on the ground, a system would need to incorporate methods for dealing with both simultaneous scheduling and limited surveillance range for arrival aircraft.

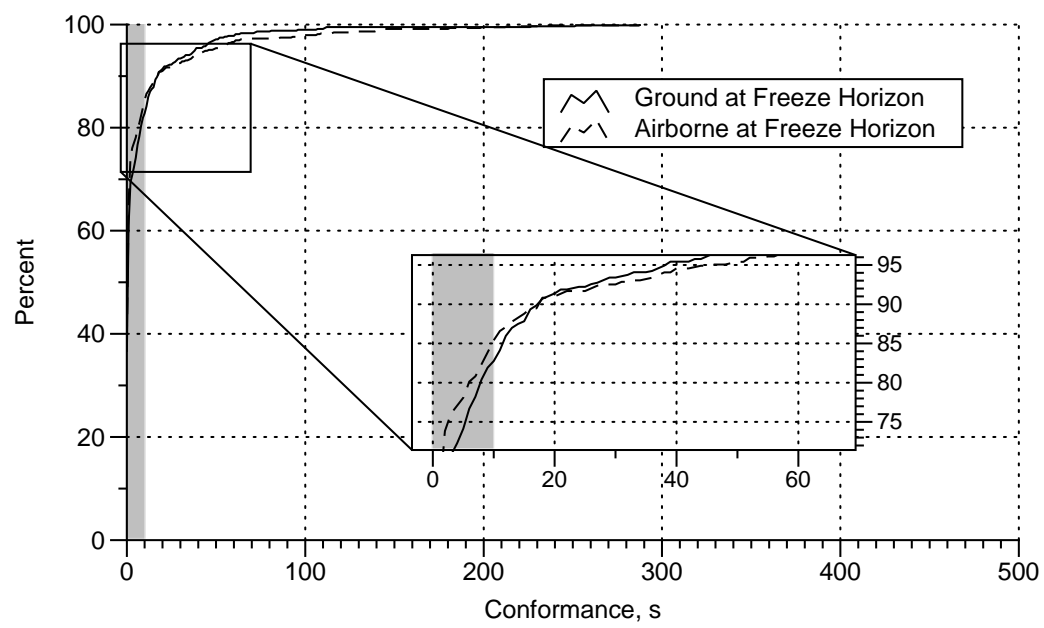

(a)

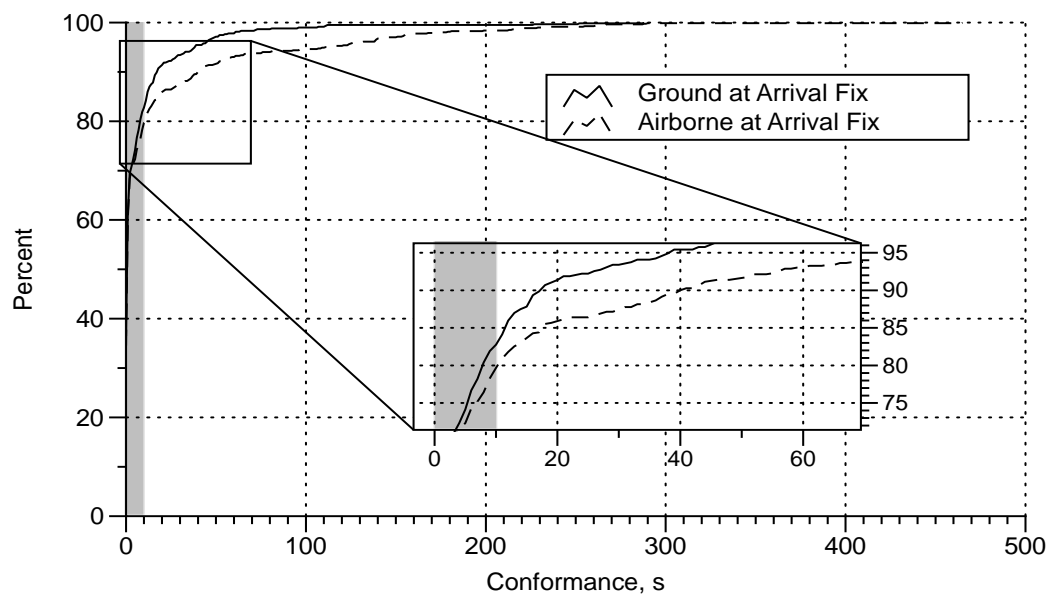

(b)

Figure 5. CDF of Separated Scheduler conformance (a) Freeze horizon (b) Arrival fix 
Table 1 categorizes the cases in the simulations for the aggregate of the three days of when aircraft needed to maneuver again after the freeze horizon. The rates of these are dependent on parameters such as surveillance range and trajectory update cycle. As a reminder, the surveillance range was $100 \mathrm{nmi}$ and the update cycle was 30 seconds in these simulations. This table shows that overall, these cases occurred in $3.8 \%$ of all arrivals in the integrated system and $2.8 \%$ in the separated system. This means that in the integrated system $2 \%$ of all arrivals were forced to maneuver again after the freeze horizon because of surveillance range limitations, and $1.8 \%$ because of simultaneous scheduling. For the separated system, $0.9 \%$ of arrivals were forced to maneuver again after the freeze horizon due of surveillance range limitations and $1.9 \%$ due to simultaneous scheduling. These results highlight the different effect of surveillance range limitations on the two scheduling concepts for airborne systems. For the integrated system, the airborne scheduling agent has no information about any other aircraft outside its surveillance range. In the separated system, however, the ground scheduling agent is generating a schedule for all arrivals, so the aircraft attempting to create scheduling maneuvers for itself has access to the STAs of the other arrivals, even if they are too far away to send their trajectory information. This lack of coordination between aircraft when they are generating maneuvers needs to be addressed when designing an airborne concept.

Table 1. Percentage of aircraft that needed to maneuver again after the freeze horizon

\begin{tabular}{|l|l|l|}
\hline & Integrated system & Separated system \\
\hline$\%$ (Total cases from total arrivals) & 3.8 & 2.8 \\
\hline$\%$ (Surveillance from total arrivals) & 2.0 & 0.9 \\
\hline$\%$ (Simultaneous scheduling from total arrivals) & 1.8 & 1.9 \\
\hline
\end{tabular}

Figure 6 shows for the integrated and separated system, the total delay for all arrivals in seconds categorized by the cause of the delay. In the integrated system, the total delay for arrivals was 597 minutes (35,820 seconds) for airborne compared to 526 minutes (31,560 seconds) for the ground. In the separated system, the delay was 559 minutes (33,540 seconds) for airborne and 521 minutes $(31,260$ seconds) for the ground. Scheduling maneuvers (i.e. maneuvers executed solely to meet an STA) at the freeze horizon made up the vast majority of total delay accrued between freeze horizon and arrival fix. The ground-based system does not accumulate any delay after the freeze horizon. It has perfect trajectory information for all aircraft and does not need to adjust the trajectories of aircraft after they have been scheduled. For the airborne system, aircraft that executed scheduling maneuvers simultaneously due to lack of coordination contributed the majority of delay accrued after the freeze horizon. The other major source of delay was due to maneuvers that were needed to avoid aircraft detected after the freeze horizon due to surveillance range limitations.

The delay at the freeze horizon for the airborne system is lower than that for the ground-based system most likely because there is no coordination between aircraft. Thus, the airborne agents can select maneuvers with less delay that will meet the schedule without realizing that those trajectories are not conflict free. The ground system, however, has knowledge of all aircraft trajectories and thus can detect all potential conflicts while choosing a scheduling maneuver. In the integrated system, a larger percentage of the delay is caused by surveillance range than in the separated system. This is because each arrival aircraft in the integrated system has no knowledge of any aircraft outside its detection range and thus will not receive information on all the STAs of aircraft currently in the arrival stream. This means that two aircraft might try to schedule themselves for the same time slot, forcing one of those aircraft to reschedule after the freeze horizon. However in the separated system, even if another aircraft is outside the $100 \mathrm{nmi}$ range, its scheduled time is known. The separated system also has lower total delay than the integrated system in the airborne cases. This is also due to the reduced impact of surveillance range limitations for the separated system. 


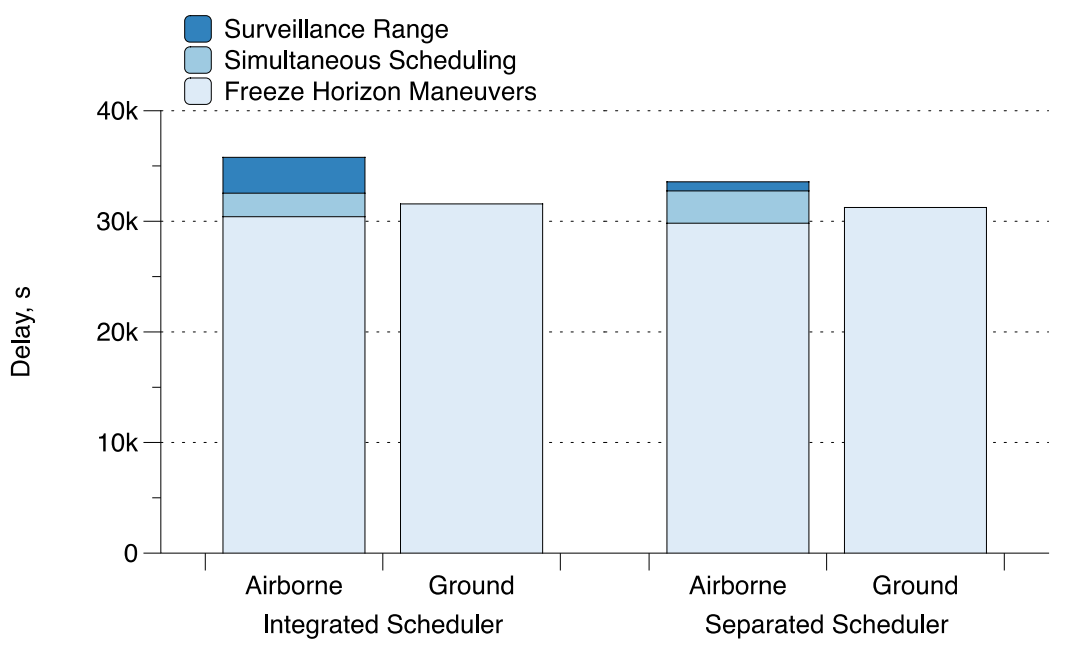

Figure 6. Sources of delay between freeze horizon and arrival fix

Fig. 7 shows the desired versus actual delay for both the ground and airborne systems with separated scheduling. The integrated systems use feedback to match the target scheduled time to the aircraft's predicted time, so this comparison of delays is not relevant. The desired delay is the delay required for an aircraft to conform to its STA. The actual delay is the flight time difference between an aircraft's original, unscheduled trajectory, and the trajectory it actually flew from the freeze horizon to the arrival fix.

The ideal curve in both of these cases is a diagonal line through the origin with a slope of one. This represents a perfect match between desired and actual delay. There are a few main points to note in these plots. Firstly, there are no actual delays more than two seconds under the desired delay. This is important for maintaining safety as receiving less than the required delay could cause an aircraft to cross the arrival fix too close to the aircraft ahead of it. Secondly, there are negative delays in this system. There are times when it is desirable for an aircraft to speed up in order to make a potential scheduling slot. This can help alleviate overall system delays, but it requires a system that can implement negative delays. It is important, for example, that the maneuvers required to meet these slots are flyable and safe from the aircraft's perspective. That, in turn, requires a good understanding of aircraft performance profiles. Otherwise, it will be very difficult to create the time-saving maneuvers required to take advantage of those potential slots.

Comparing the two plots, there are a few differences that are worth mentioning. The ground system has more data points close to the "ideal" line than the airborne system. This implies that the ground-based system had an easier time meeting the required delays. As the underlying algorithm for searching for maneuvers is virtually identical, the reasons for these differences are once again due to the surveillance range value used in these simulations and the effect of simultaneous arrivals. Previous figures showed that these post-freeze horizon maneuvers reduced scheduling conformance and increased delay. Figure 7 shows how that extra delay was accumulated. In this case, it is clear that much of the extra delay is in aircraft that originally required very modest delays. Most of the aircraft that were multiple minutes above the ideal line had a desired delay of less than three minutes. The airborne case also has more aircraft that had an actual delay even though their desired delay was zero or near zero.

These results could differ with a different implementation of priority scheduling. If scheduling order was to be maintained at all cost, for example, one would expect to see many more cases where aircraft drifted above their originally desired delay, but there might not be as many cases where the actual delay was many minutes higher than the desired. However, the point of these plots is simply to highlight that the airborne system has a more complicated problem, depending on the constraints and system design, that requires extra attention be given to aircraft that need to reschedule inside the freeze horizon. 


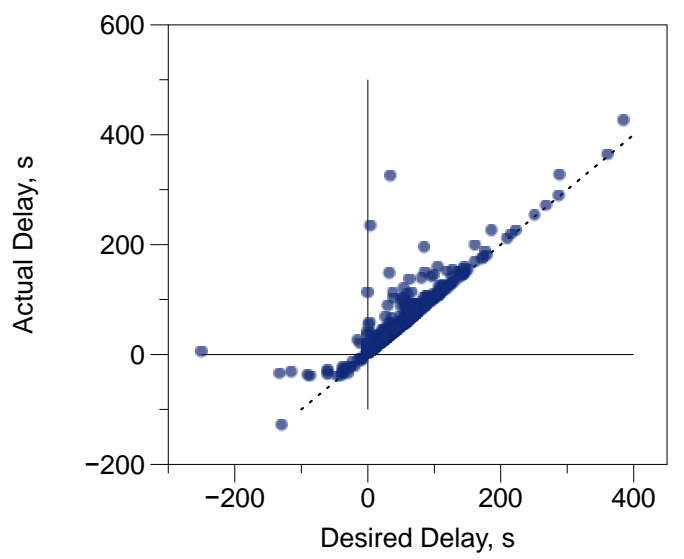

(a)

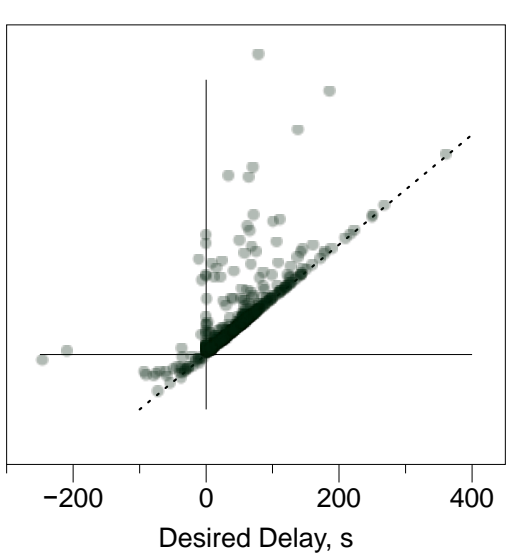

(b)

Figure 7. Desired vs. Actual Delay (a) Ground (b) Airborne

\section{Conclusion}

In a system with no uncertainty and complete trajectory intent data, both airborne and ground systems were able to schedule and fly approximately nine hours of current traffic taken for three different days into a simplified KDFW TRACON without losses of separation. Two different scheduling algorithms were used. One was integrated into the scheduling conformance and CD\&R system, and was located on the ground for the ground system and in the aircraft for the airborne system. The other scheduler was separated, and always located on the ground while the scheduling conformance and CD\&R systems could be either on the ground or on the aircraft. A surveillance range limitation of $100 \mathrm{nmi}$ and a time delay for sharing updated trajectory intent (one cycle or 30 seconds) were implemented for the airborne system. These parameters had the effect of forcing $3.8 \%$ of scheduled arrivals to break their schedule for the integrated airborne system, and $2.8 \%$ for the separated airborne system. Both airborne scheduling systems had a similar number of broken schedules due to aircraft scheduling themselves simultaneously. However, in the separated case, $2 \%$ of scheduled arrivals were forced to maneuver again after the freeze horizon due to surveillance range limitations versus $0.9 \%$ of scheduled arrivals in the separated system. Different flight rules, data sharing update rates, and surveillance range will likely change the percentages, but the underlying factors will remain. However, it is worth noting that even with the limitations enforced on the airborne system, all aircraft landed safely. In the integrated system, the total delay for arrivals was 597 minutes for airborne compared to 526 minutes for the ground. In the separated system, the delay was 559 minutes for airborne and 521 minutes for the ground.

It should be noted that using different concepts for data sharing and decision making could change the relative importance of these metrics. These results could also change with increased traffic demand, as higher density arrival flows may have different impacts on different configurations. These are both areas of possible future study.

Overall, these results indicate that the surveillance range and the sharing of trajectories and STAs are important factors in determining the efficiency of an airborne arrival management system. Creating a schedule external to the CD\&R and scheduling conformance system was seen to reduce total delays for the airborne system, and had a minor effect on the ground. The effect of an external scheduler on other metrics was mixed. Further study of airborne arrival concepts with more realistic trajectories and more detailed operational concepts is required before these systems could be declared feasible. A logical extension of this work would be to include trajectory prediction errors, which will better test the robustness and safety of the system. 


\section{Acknowledgments}

The authors would like to thank Todd Lauderdale, David Thipphavong, and Nelson Guerreiro for their valuable input. Special thanks to Chok Fung "Jack" Lai and Tony Wang for software development and Jeff Piety, Luis Vargas, and Justin Schrout for their help with the data analysis tools.

\section{References}

${ }^{1}$ Erzrberger, H., "Automated Conflict Resolution for Air Traffic Control," 25th International Congress of the Aeronautical Sciences, Germany, 2006.

${ }^{2}$ NASA. Concept definition for distributed air/ground traffic management (dag-tm). In Advanced Air Transportation Technologies (AATT) Project, Aviation System Capacity (ASC) Program, volume Version 1.0, September 301999.

${ }^{3}$ Wing, D.J. and Cotton, W.B. Autonomous Flight Rules - a concept for self-separation in U.S. domestic airspace. NASA/TP-2011-217174, 2011.

${ }^{4}$ Bilimoria, K. D., "Survey of Air/Ground and Human/Automation Functional Allocation for Separation Assurance," 12th AIAA Aviation Technology, Integration, and Operations (ATIO) Conference and 14th AIAA/ISSM. Indianapolis, IN, 2012.

${ }^{5}$ Wing, D., et al., "Comparison of Groundbased and Airborne Function Allocation Concepts for NextGen."

${ }^{6}$ Letsu-Dake, E., Rogers, W., Dorneich, M., and De Mers, R. "Innovative Flight Deck Function Allocation Concepts for NextGen," In Advances in Human Aspects of Aviation, S.J. Landry ed., CRC Press, Boca Raton, FL, 2012, pp. 301-310.

${ }^{7}$ Parasuraman, R., Sheridan, T., and Wickens, C. D., "A Model for Types and Levels of Human Interaction with Automation," IEEE Transactions on Systems, Man and Cybernetics, Part A: Systems and Humans, Vol. 30, No. 3, May 2000.

${ }^{8}$ Techakesari, O., and Ford, J. J., “Automated centralised separation management with onboard decision support," 27th International Congress of the Aeronautical Sciences (ICAS), ICAS2010-11.7.3, September 2010.

${ }^{9}$ Battiste, V., et al., "Distributed Air/Ground Traffic Management-Technology and Concept Demonstration Report," AIAA Aircraft Technology, Integration, and Operations (ATIO), Los Angeles, CA, October 2002.

${ }^{10}$ Hoekstra, J., van Gent, R., and Ruigrok, R., "Conceptual Design of Free Flight with Airborne Separation Assurance," NLR-TP-98252, 1998.

${ }^{11}$ Wing, D., et al., "Pilot and Controller Evaluations of Separation Function Allocation in Air Traffic Management," 10th USA/Europe ATM 2013 R\&D Seminar, 2013.

${ }^{12}$ Sokkappa, B.G., "The Impact of Metering Methods on Airport Throughput,” MITRE MP-89W000222, 1989.

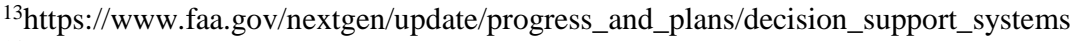

${ }^{14}$ Wong, G., "The Dynamic Planner: The Sequencer, Scheduler, and Runway Allocator for Air Traffic Control Automation," NASA TM-2000-209586, 2000.

${ }^{15}$ Swenson, H. N., et al., "Design and Operational Evaluation of the Traffic Management Advisor at the Fort Worth Air Route Traffic Control Center," 1st USA/Europe Air Traffic Management Research \& Development Seminar, Saclay, France, June 17 - 19, 1997.

${ }^{16}$ Aweiss, A. S., Farrahi, A. H., Lauderdale, T. A., Thipphavong, A. S., and Lee, C. H., "Analysis of a Real-Time Separation Assurance System with Integrated Time-in-Trail Spacing," 10th AIAA Aviation Technology, Integration, and Operations Conference, Fort Worth, TX, 2010.

${ }^{17}$ Aweiss, A. S., Lauderdale, T. A., "Evaluation of Integrated Arrival Scheduling and Automated Conflict Detection and Resolution System," 12th AIAA Aviation Technology, Integration, and Operations Conference, Indianapolis, IN, 2012.

${ }^{18}$ Erzberger, Heinz; Transforming the NAS: The Next Generation Air Traffic Control System; Proceedings of the International Congress of the Aeronautical Sciences (ICAS), August 30, 2004, Yokohama, Japan.

${ }^{19}$ Erzberger, H., "Separation Assurance in the Future Air Traffic System," ENRI International Workshop on ATM/CNS, Tokyo, Japan, 2009.

${ }^{20}$ Erzberger, H., Lauderdale, T. A., Chu, Y. C., “Automated Conflict Resolution, Arrival Management and Weather Avoidance for ATM," 27th International Congress of the Aeronautical Sciences (ICAS), Nice, France, 2010.

${ }^{21}$ Holladay, J., Lewis, T., Lauderdale, T. A., "Modeling and Simulation of Function Allocation Concepts for Separation Assurance," International Conference on Human-Computer Interaction in Aerospace, Santa Clara, CA, 2014.

${ }^{22}$ Chung, W.W., and Staab, R., “A 1090 Extended Squitter Automatic Dependent Surveillance - Broadcast (ADSB) Reception Model for Air-Traffic-Management Simulations", Proceedings of AIAA Modeling and Simulation Technologies Conference and Exhibit, AIAA-2006- 6614.

${ }^{23}$ RTCA, "Minimum Performance Standards for $1090 \mathrm{MHz}$ Extended Squitter Automatic Dependent Surveillance Broadcast (ADS-B) and Traffic Information Services - Broadcast (TIS-B),”DO-260B, 2009.

${ }^{24}$ Farley, T., Kupfer, M., and Erzberger, H., "Automated Conflict Resolution: A Simulation Evaluation Under High Demand Including Merging Arrivals," 7th AIAA Aviation Technology, Integration and Operations Conference, Belfast, Northern Ireland, 2007. 
${ }^{25}$ Kupfer, M., Farley, T., Chu, Y. C., and Erzberger, H., “Automated Conflict Resolution: A Simulation-Based Sensitivity Study of Airspace and Demand," 26th International Congress for the Aeronautical Sciences, Anchorage, AK, 2008.

${ }^{26}$ Farley, T. Erzberger, H., "Fast-Time Simulation Evaluation of A Conflict Resolution Algorithm Under High Air Traffic Demand," 7th USA/Europe ATM R\&D Seminar, Barcelona, Spain, 2007. 